\title{
Analysis of Vagina Microecology and Investigation of Common Infection in Border Area of Chongzuo City
}

\author{
Huan Zhao*\#, Yan Chen*, Liuxian Huang, Tianyou Huang, Miaohong Li, Wanwei Yang, Qing Peng, \\ Lili Yu, Haisha Wei, Jianlan Gan, Xiuge Li
}

The Clinical Laboratory of Chongzuo Maternal and Child Health Care Hospital, Chongzuo, China

Email:"122610256@qq.com

How to cite this paper: Zhao, H., Chen, Y., Huang, L.X., Huang, T.Y., Li, M.H., Yang, W.W., Peng, Q., Yu, L.L., Wei, H.S., Gan, J.L. and Li, X.G. (2022) Analysis of Vagina Microecology and Investigation of Common Infection in Border Area of Chongzuo City. Advances in Reproductive Sciences, 10, 1 11.

https://doi.org/10.4236/arsci.2022.101001

Received: January 12, 2022

Accepted: February 11, 2022

Published: February 14, 2022

Copyright $\odot 2022$ by author(s) and Scientific Research Publishing Inc. This work is licensed under the Creative Commons Attribution International License (CC BY 4.0).

http://creativecommons.org/licenses/by/4.0/

\begin{abstract}
Objective: To investigate the microecology and common infection of the female vagina in border area of Chongzuo City. Methods: Female patients in the outpatient department and inpatient department of gynecology of our hospital from April 1, 2018 to August 23, 2021 were selected as the research objects. The reproductive tract secretions of all patients were examined by microecological examination, morphological examination, chemical analysis and microscopic examination, and the results of various indicators were statistically analyzed to draw relevant conclusions. Results: Among the 1498 women with vaginal microecological imbalances, cleanliness was determined to be 534 cases of degree II, 868 cases of degree III, and 96 cases of degree IV; Nugent scores were 9 - 10 in 9 cases, 8 in 122 cases, 7 in 30 cases, 6 in 18 cases, 5 in 8 cases, 4 in 717 cases, 3 in 248 cases, 2 in 209 cases, 1 in 118 cases, and 0 in 19 cases. AV scores were 8 in 1 case, 7 in 1 case, 6 in 2 cases, 5 in 9 cases, 4 in 84 cases, 3 in 207 cases, 2 in 850 cases, 1 in 284 cases, and 0 in 60 cases. Among the pathogen infections, 99 cases were mycoplasma, 361 cases were candida, 199 cases were bacterial and 8 cases were trichomonas; In chemical analysis, there were 138 cases with pH 5.1, 326 cases with $\mathrm{pH} 4.8,775$ cases with $\mathrm{pH}$ 4.6, 217 cases with $\mathrm{pH}$ 4.4, and 42 cases with pH 4.1; meanwhile, 1390 cases were positive for hydrogen peroxide, 505 cases were positive for glucosidase, 487 cases were positive for acetylglucosaminidase, 184 cases were positive for sialidase, 290 cases were positive for proline aminopeptidase, 501 cases were positive for coagulase, and 1064 cases were positive for leukocyte esterase. Conclusion: Female reproductive tract microecology is more complex, and the pathogens of infection are also diverse. A systematic examination can clarify the occurrence and development of the disease, and then provide reliable laboratory data for clinical treatment and guide clinical medication.
\end{abstract}




\section{Keywords}

Border Area, Chongzuo City, Female, Reproductive Tract, Microecology, Infection

\section{Introduction}

A variety of microorganisms are parasitized in the female vagina. Under normal circumstances, the micro-ecology maintains the stable balance; when the microecological balance is disrupted, pathogenic microorganisms will continue to breed and cause vagina-related diseases [1] [2] [3]. In recent years, the examination of vaginal secretions is mainly based on microscopy which has high experience requirements and strong subjectivity; and the inspection process is affected by the quality of the microscope and the uniformity of the smear; moreover, diagnosis only by morphological identification has certain defects in sensitivity and specificity, which is difficult to meet the needs of clinical diagnosis [4] [5] [6]. This situation further leads to the low cure rate, high recurrence rate and low rational use rate of antibiotics in the clinical treatment of gynecological infection, which brings many unnecessary troubles to doctors and patients [7] [8]. Vaginal microecology analysis can assist in the diagnosis of women's vaginal health, including microecological environment, vaginitis, bacterial vaginosis (BV), trichomonas vaginitis, vulvovaginal candidiasis; vaginal microecology analysis can also be used for vaginal microecology assessment of women, auxiliary diagnosis of suspicious vaginal infections, finding asymptomatic infections, investigating cases of drug resistance caused by conventional treatment, helping clinical selection of treatment options, judging the recovery, and carrying out prevalence survey of vaginal infection, etc [9] [10] [11] [12]. In order to understand the microecology and common infection of the female reproductive tract in Chongzuo City, the enrolled patients were analyzed. The results are reported as follows.

\section{Materials and Methods}

\subsection{Research Objects}

A total of 1498 female patients were enrolled from April 1, 2018 to August 23, 2021 in the outpatient department and inpatient department of gynecology of our hospital. They were between 7 and 74 years old, with an average age of 33.45 \pm 9.22 years. All cases felt vaginal discomfort and came to see a doctor, excluding other related diseases. The cases used in this study were signed by the patients themselves and approved by the medical ethics committee of our hospital.

\subsection{Method}

\subsubsection{Specimen Collection}

Collected by the clinician, a sterile swab or cotton swab is dipped in sterile normal saline and inserted into the patient's vagina, and the dome is rotated 3 - 5 
times, and placed in a sterile plastic test tube for inspection.

\subsubsection{Instruments and Reagents}

The equipment uses the Shanghai Xingyao company ACT-2000 ultra-high magnification microscopy system, Antu AutowoMO automatic genital secretion workstation, Deere heater, Deere automatic staining machine DL-DYE-GR-8, sterile swab or cotton swabs and supporting reagents are provided by Antu Company.

\subsubsection{Detection Method}

1) Take the kit out of the refrigerated environment and place it at room temperature $\left(18^{\circ} \mathrm{C}-25^{\circ} \mathrm{C}\right)$ to equilibrate for at least 30 minutes; 2) Sample processing: Take a sample adding test tube, drop the joint test II diluent to between the two graduation lines at the bottom of the test tube, place the collected vaginal secretion swab in the test tube, wash it thoroughly and gently squeeze it with your fingers On the test tube wall, make the liquid absorbed by the swab flow back to the test tube, discard the swab, and get the sample liquid: A. Suck the sample solution through a straw, add 1 drop of sample solution (about $30 \mathrm{ul}$ ) to a clean slide, make thin slices, dry them in a Deere heater at $75^{\circ} \mathrm{C}$, and then use DL-Dye-GR-8 automatic Deere dyeing machine for Gram staining; After that, the microecological evaluation system indexes (epithelial cells, density of bacteria, diversity of bacteria, proportion of Gram-positive slender bacillus and proportion of other bacteria) were completed in ultrahigh magnification microscopic system; B. Use a straw to absorb the sample solution, add 1 drop of sample solution (about $30 \mathrm{ul}$ ) to the clean glass slide to make the wet slide, and complete the morphological examination of the wet slide (red blood cells, clue cells, cleanliness, white blood cells, pus cells, Trichomonas, mycoplasma, chlamydia, intracellular G-diplococcus and other indicators) in the ultrahigh power microscopic system. C. Take out the joint inspection card from the package, gently remove the plate stick, and use a straw to absorb the sample liquid. Add 1 drop of sample liquid to each of the first five holes of the joint inspection card, each drop is about $30 \mathrm{ul}$; 3) Put the five couplet detection card into Antu autowomo automatic reproductive tract secretion workstation for detection, judge with software, and score in strict accordance with $\mathrm{Nu}$ gent and BV scoring standards. The results are transmitted to the information system and the inspection report is printed.

\subsubsection{Result Judgment}

1) The first hole (hydrogen peroxide): no color or light yellow (marked as "+") is positive, indicating imbalance of vaginal flora, pathological or sub-healthy vaginal environment; Light red (marked " \pm "), indicating the presence of moderate lactobacillus; 2) (Leukocyte esterase): no color or light color (marked with “-”) indicates negative, indicating The second hole that the leukocytes are less than $5 / \mathrm{HP}$; light blue (marked with " \pm ") indicates negative, indicating that leukocytes $5 \sim 15 / \mathrm{HP}$; blue (marked as “+”, “++”, “+++”) indicates positive, indicating that the white blood cells are greater than 15/HP; 3 ) The third hole (neuraminidase): 
no color or orange (marked with "-") indicates negative; light red (marked with “ \pm ") indicates weak positive; red, purple, blue, brown or black (marked with “+”) indicates a positive hole that is positive for bacterial vaginosis; 4) The fourth hole (proline aminopeptidase): no color or light color (marked with “-”) indicates negative; light yellow (marked with " \pm ") indicates weak positive; yellow (marked with “+”) means positive. If the hole is positive at the same time as the $5^{\text {th }}$ hole is negative, it indicates $\mathrm{BV}$ infection. If the $5^{\text {th }}$ hole is positive at the same time, the result will be judged according to the $5^{\text {th }}$ hole; 5 ) The fifth hole (acetylglucosaminidase): no color or light color (marked with “-”) indicates negative; light yellow (marked with " \pm ") indicates weak positive; yellow (marked with " + ") indicates positive. If the hole is positive at the same time $\mathrm{Ph} \geq 4.8$, it indicates trichomoniasis vaginitis, and if the hole is positive at the same time $\mathrm{pH} \leq 4.6$, it indicates vulvovaginal candida infection; 6) The sixth hole (PH): the color changes from yellow $\rightarrow$ cyan $\rightarrow$ green $\rightarrow$ blue, indicating that the $\mathrm{pH}$ changes from 3.8 to 5.4. Compared with the five joint test color card of vaginitis, the green $\mathrm{pH}$ is $\leq 4.6$ and the blue $\mathrm{pH}$ is $\geq 4.8$.

\subsubsection{Interpretation of Inspection Results}

1) For suspicious specimens with weakly positive results detected by this kit, re-determination and dynamic observation are recommended; 2) Due to methodological or specific reasons, different results may be obtained when the same sample is tested with reagents from different manufacturers. Therefore, the results obtained from different reagents should not be directly compared with each other to avoid false medical interpretation.

\subsection{Explanation of Relevant Scores}

Nugent score was an index to examine the microecology of vagina. Bacterial morphology was observed by gram staining and oil microscope, and lactobacillus, Gardnerella, Prevobacterium and Mobiluncus were scored by semi-quantitative evaluation method. The sum of the morphological values of the above 4 kinds of bacteria is the total value. If there are no miscellaneous bacteria, the score is zero, which means that there is no bacterial vaginosis. The more miscellaneous bacteria, the higher the score, and the greater the possibility of bacterial vaginosis. The normal range is $0-3$ points, BV: Nugent score $\geq 7$ points, BV intermediate: Nugent score 4 - 6 points, if the score is higher than 3 points, bacterial vaginosis is considered, and timely treatment is recommended. The AV score includes five detection indicators: LBG grade, white blood cell count, percentage of white blood cells containing toxic particles, background colonies, and percentage of parabasal epithelial cells. The score of each indicator can be divided into normal or mild (0 points), moderate (1 point) and severe ( 2 points), the scores of each indicator are summed up to obtain the total AV score. Diagnostic criteria: AV score $<3$ points, no AV signs; AV score 3 - 4 points, mild AV. The routine inspection report of vaginal discharge generally includes the inspection of cleanliness, mold and trichomoniasis. 


\subsection{Statistical Analysis}

Statistical analysis was performed using statistical software SSPS 24.0. Data statistics were expressed as $(\mathrm{x} \pm \mathrm{s})$, the comparison of the number of cases of vaginosis was performed by the chi-square test, and the comparison of the mean data between groups with normal distribution was performed by the $t$ test. $\mathrm{P}<0.05$ was considered to be statistically significant.

\section{Results}

Among the 1498 women with vaginal microecological imbalances, 534 cases were classified as grade II, accounting for 35.65\%; 868 cases were grade III, accounting for $57.94 \%$, and 96 cases were grade IV, accounting for $6.41 \%$; Nugent score of 9 - 10 points in 9 cases, 8 points in 122 cases, 7 points in 30 cases, 6 points in 18 cases, 5 points in 8 cases, 4 points in 717 cases, 3 points in 248 cases, 2 points in 209 cases, 1 point in 118 cases, 0 point in 19 cases; 8 points of AV score in 1 case, 7 points in 1 case, 6 points in 2 cases, 5 points in 9 cases, 4 points in 84 cases, 3 points in 207 cases, 2 points in 850 cases, 1 point in 284 cases, and 0 points in 60 cases; Among the pathogen infections, there were 99 cases of Mycoplasma infection, accounting for $6.61 \%$; 361 cases of Candida infection, accounting for $24.10 \%$; 199 cases of bacterial infection, accounting for $13.28 \%$, and 8 cases of trichomonas infection, accounting for $0.53 \%$; In chemical analysis, 138 cases with $\mathrm{PH}$ value of 5.1, accounting for 9.21\%, 326 cases with $\mathrm{PH}$ value of 4.8, accounting for $21.76 \%, 775$ cases with $\mathrm{PH}$ value of 4.6 , accounting for $51.74 \%$, 217 cases with $\mathrm{PH}$ value of 4.4 , accounting for $14.49 \%$, and 42 cases of PH4.1, accounting for $2.80 \%$; Hydrogen peroxide was positive in 1390 cases, accounting for $92.79 \%$, negative in 108 cases, accounting for $7.21 \%$; glucosidase positive in 505 cases, accounting for $33.71 \%$, negative in 993 cases, accounting for $66.29 \%$; acetylglucosidase positive in 487 cases, accounting for $32.51 \%, 1011$ cases were negative, accounting for $67.49 \%$; 184 cases were sialidase positive, accounting for $12.28 \%, 1314$ cases were negative, accounting for $87.72 \%$; 290 cases were proline aminopeptidase positive, accounting for $19.36 \%$, and 1208 cases were negative, accounting for 80.64\%; Coagulase positive in 501 cases, accounting for $33.44 \%$, negative in 997 cases, accounting for 66.56\%; leukocyte esterase positive in 1064 cases, accounting for $71.03 \%$, negative in 434 cases, accounting for $28.97 \%$. The specific results are shown in Tables 1-5.

\section{Discussion}

Female Genital Tract (FGT) is an open cavity and is an important microecological settlement area of human body. Under normal circumstances, the vaginal microecological environment is composed of four parts of various vaginal flora, periodic endocrine changes, normal vaginal anatomy and vaginal local immune system [13][16]. When the vaginal flushing, menstruation, contraceptives, sexual intercourse frequency and long-term use of immunosuppressors, broad-spectrum antibiotics, 
Table 1. Comparison of cleanliness in 1498 female patients with vaginal microecological imbalance.

\begin{tabular}{ccc}
\hline Cleanliness & Number of cases & Percentage (\%) \\
\hline II degree & 534 & 35.65 \\
IIIdegree & 868 & 57.94 \\
IVdegree & 96 & 6.41 \\
\hline
\end{tabular}

Notes: Comparison of cleanliness class II and class III, $\chi^{2}=149.5541, \mathrm{P}<0.0000$; the comparison between degree II and degree IV, $\chi^{2}=385.5980, \mathrm{P}<0.0000$; the comparison between degree III and degree IV, $\chi^{2}=911.5399, \mathrm{P}<0.0000$.

Table 2. Correlation score comparison of 1498 female patients with vaginal microecological imbalance.

\begin{tabular}{ccccc}
\hline Score & $\begin{array}{c}\text { Nugent score } \\
\text { (cases) }\end{array}$ & $\begin{array}{c}\text { Percentage } \\
(\%)\end{array}$ & $\begin{array}{c}\text { AV score } \\
\text { (Cases) }\end{array}$ & $\begin{array}{c}\text { Percentage } \\
(\%)\end{array}$ \\
\hline 9 - 10 Points & 9 & 0.60 & 0 & 0.00 \\
8 Points & 122 & 8.14 & 1 & 0.07 \\
7 Points & 30 & 2.00 & 1 & 0.07 \\
6 Points & 18 & 1.20 & 2 & 0.13 \\
5 Points & 8 & 0.53 & 9 & 0.60 \\
4 Points & 717 & 47.86 & 84 & 5.61 \\
3 Points & 248 & 16.56 & 207 & 13.82 \\
2 Points & 209 & 13.95 & 850 & 56.74 \\
1 Points & 118 & 7.88 & 284 & 18.96 \\
0 Points & 19 & 1.27 & 60 & 4.01 \\
\hline
\end{tabular}

Notes: Nugent A score of 0 - 3 is normal; If the score is greater than 3, bacterial vaginosis is considered; AV score $<3$ points, no AV signs; AV score 3 - 4 points, mild AV.

Table 3. Comparison of pathogen infection in 1498 female patients with vaginal microecological imbalance.

\begin{tabular}{ccc}
\hline Pathogen & Number of cases & Percentages (\%) \\
\hline Mycoplasma & 99 & 6.61 \\
Candida & 361 & 24.10 \\
Bacteria & 199 & 13.28 \\
Trichomonad & 8 & 0.53
\end{tabular}

Note: comparison between mycoplasma infection and Candida infection, $\chi^{2}=176.2939$, $\mathrm{P}<0.0000$; Compared with mycoplasma infection and bacterial infection, $\chi 2=37.2635$, $\mathrm{P}<0.0000$; Comparison between mycoplasma infection and Trichomonas infection, $\chi^{2}=$ 80.2589, $\mathrm{P}<0.0000$; Candida infection compared with bacterial infection, $\chi^{2}=57.6377, \mathrm{P}$ $<0.0000$; Comparison between Candida infection and Trichomonas infection, $\chi^{2}=385.1277$, $\mathrm{P}<0.0000$; Comparison between Bacterial infection and Trichomonas infection, $\chi^{2}=$ 189.3170, $\mathrm{P}<0.0000$. 
Table 4. Comparison of $\mathrm{pH}$ in 1498 female patients with vaginal microecological imbalance.

\begin{tabular}{ccc}
\hline PH value & Number of cases & Percentage (\%) \\
\hline 5.1 & 138 & 9.21 \\
4.8 & 326 & 21.76 \\
4.6 & 775 & 51.74 \\
4.4 & 217 & 14.49 \\
4.1 & 42 & 2.80 \\
\hline
\end{tabular}

Note: $\mathrm{pH} \geq 4.8$ indicates trichomonal vaginitis, and $\mathrm{pH} \leq 4.6$ indicates vulvovaginal Pseudofilament yeast infection.

Table 5. Comparison of chemical analysis results of 1498 female patients with vaginal microecological imbalance.

\begin{tabular}{ccccc}
\hline Project & Positive (cases) & Negative (cases) & $\chi^{2}$ Value & P value \\
\hline Hydrogen peroxide & 1390 & 108 & 2194.2911 & 0.0000 \\
Glucosidase & 505 & 993 & 317.9493 & 0.0000 \\
Acetyl glucosaminidase & 487 & 1011 & 366.5901 & 0.0000 \\
Neuraminidase & 184 & 1314 & 1704.8064 & 0.0000 \\
Proline aminopeptidase & 290 & 1208 & 1125.1322 & 0.0000 \\
Clotting enzyme & 501 & 997 & 328.4593 & 0.0000 \\
White cell esterase & 1064 & 434 & 529.9065 & 0.0000 \\
\hline
\end{tabular}

corticoids and other endogenous and exogenous factors, vaginal microecological environment will change which cause vaginal flora disorders and a large number of conditional pathogenic bacteria reproductive infection, leading to the occurrence of vaginitis.

Female genital tract infection is a common and frequently occurring disease in gynecological diseases [17] [18] [19] [20]. It is mainly manifested in peculiar smell, increase of vaginal secretions, pruritus of vulva, swelling of lower abdomen, burning sensation and other symptoms. It can be caused by bacteria, fungi, gonococcus, Trichomonas vaginalis, mycoplasma, chlamydia and other conditional pathogens. If it is not diagnosed and treated in time, it can cause cervicitis, cervical erosion Vaginitis, pelvic inflammatory disease, premature delivery, infertility and other complications; severe cases can also increase the risk of cervical cancer and AIDS infection. The physical and mental health of women of childbearing age has been seriously affected, which is a major global social problem and public health problem.

According to statistics, there are about 1 billion outpatient visits in obstetrics and gynecology in China every year, of which about 500 million have vaginal infections, and recurrent infections account for about 50\%, resulting in infertility of about 30 million women of childbearing age every year, premature rupture of 
membranes or even premature delivery of 3 million pregnant women, and the annual cost of medical treatment is as high as 40 billion Yuan. It is the number one killer of women's health in China [21] [22]. Female genital tract infection needs to be clear pathogen infection in order to symptomatic treatment and restore vaginal microecological balance.

With the continuous development and progress of inspection technology, the application of vaginal secretion zymography analysis technology is more and more widely, and its functional enzyme index combined with routine vaginal secretion microscopy can quickly and comprehensively reflect the existence of various inflammations and microecology in the vagina which can provide a basis for the diagnosis of vaginal inflammation types, so as to better guide clinical diagnosis and treatment, and improve the current status of diagnosis and treatment of female reproductive tract diseases [23] [24] [25].

The results of this study showed that in 1498 women with vaginal microecological imbalances, the cleanliness accounted for $64.35 \%$ between grades III and IV, and the differences were statistically significant in the pairwise comparison of cleanliness, $\mathrm{P}<0.001$. It suggests that the incidence of vaginitis is relatively high in women with vaginal microecological imbalance; There were 904 cases with Nugent score greater than 3, accounting for $60.34 \%$, It was suggested that bacterial vaginitis occurred in female patients; in the AV score, 304 cases had more than mild AV signs, accounting for $20.29 \%$, suggesting that about one fifth of the cases in this group had AV signs. Among the pathogen infections, 667 cases were found to have related pathogen infections, accounting for $44.52 \%$, indicating that there was a clear pathogen infection in this group of patients; in terms of the four infectious pathogens, the pairwise comparisons showed that bacterial infections and fungal infections were more frequent; the differences were statistically significant, $\mathrm{P}<0.001$. In chemical analysis, glucosidase, Acetylglucosaminidase, sialidase, proline aminopeptidase, coagulase and leukocyte esterase were all positive in varying degrees, and the positive rates were $33.71 \%, 32.51 \%, 12.28 \%$, $19.36 \%, 33.44 \%$ and $71.03 \%$ respectively; the difference between the negative and positive results of functional enzyme test was statistically significant, $\mathrm{P}<0.001$. All the positive results suggested that there was some vaginosis in the female patients with microecological imbalance.

\section{Conclusion}

Female reproductive tract microecology is more complex, and the infection pathogens are also diverse. The systematic examination can clarify the occurrence and development of diseases, thus providing reliable laboratory data for clinical treatment and guiding clinical medication. Vaginal microecological analysis can assist in the diagnosis of vaginal health conditions in women, including microecological environment, vaginitis, bacterial vaginosis (BV), trichomonas vaginitis, and vulvovaginal candidiasis; it can be used for the evaluation of vaginal microecology in women, auxiliary diagnosis of suspected vaginal infection, finding asymptomatic infection, investigating cases with drug resistance caused by conventional treat- 
ment, helping clinical selection of treatment plan, judging recovery and investigating the prevalence of vaginal infection; it is worthy of popularization and application.

\section{Limitations of the Study}

Since the detection principle of the kit is based on biochemical reactions, if the sample is improperly collected, erroneous results can be produced. In addition, due to the complex changes in the vaginal flora of women and many interfering factors, the positive results should be diagnosed and treated in combination with clinical symptoms. In addition, the cases selected in this study are the population of Chongzuo City in the border area, and there may be differences in other areas.

\section{Acknowledgements}

During the process of this topic research, we got much help from many departments and individuals, and other personnel not involved in this project research. All of them offered great support and help in this research. Now here, all of members in this research show our deepest appreciation to them, and wish them good health and everything goes well.

\section{Funding}

The paper was supported by Guangxi Chongzuo City Scientific Research and Technology Development Project (NO. Chongke FA2018032).

\section{Conflicts of Interest}

The authors declare no conflicts of interest regarding the publication of this paper.

\section{References}

[1] Qiu, D.W., Zhong, S., Tang, X. and Zeng, X. (2021) Analysis of Vaginal Microecology and Group B Streptococcus Detection Results in 1935 Pregnant Women. Laboratory Medicine and Clinical, 18, 3274-3277.

[2] Ye, Q., Zou, L. and Cui, Y. (2021) Meta-Analysis of the Correlation between Vaginal Microecological Imbalance and Premature Rupture of Membranes during Pregnancy. Chinese Journal of Microecology, 33, 1330-1336.

[3] Chen, R., Feng, Y.Y., Feng, Z.H., Huang, Z.Y., Zhang, L., Lv, T., Zhao, M., Chen, H.Y. and Liao, Q.P. (2021) Correlation between Vaginal Microecological Abnormalities and Cervical Intraepithelial Lesions. Chinese Journal of Obstetrics and Gynecology, 22, 597-600.

[4] Wu, X.Z., Ma, F.F., Lin, L.H. and Huang, S.E. (2021) The Value of Vaginal Microecological Evaluation System to Detect Vaginal Secretions in the Diagnosis of Bacterial Vaginosis. Medical Equipment, 34, 32-33.

[5] He, S.B. and Qin, K.L. (2021) Correlation between HPV Infection in the Reproductive Tract of Women of Childbearing Age and Vaginal Microecological Characteristics. Journal of Shaoyang University (Natural Science Edition), 18, 61-66.

[6] Delinal, U. and Ma, C.L. (2021) The Relationship between Vaginal Microecological 
Imbalance and HPV Infection and Cervical Lesions. Xinjiang Medicine, 51, 11141117.

[7] Wan, Y.X. and Yang, X.W. (2021) Correlation of Vaginal Microecological Abnormalities with High-Risk Types and Human Papillomavirus Infection after AV/BV Five-Joint Examination. Xinjiang Medicine, 51, 1111-1113.

[8] Ma, X.L., Lu, Y.H., Yan, L.P., Ma, Y.Y., Chen, H., Ding, J.F., Ying, Q.H. and Wang, L.C. (2021) Analysis of Vaginal Microecological Characteristics of Patients with Polycystic Ovary Syndrome of Reproductive Age. Zhejiang Medicine, 43, 2090-2095, 2161.

[9] Han, Y., Xia, Y., Tan, Y., Hu, R.K. and Ren, Q.L. (2021) "Keep Positive and Innovation" on Wandai Decoction to Improve Vaginal Microecology in the Treatment of Vulvovaginal Candidiasis. Sichuan Traditional Chinese Medicine, 39, 30-33.

[10] Lin, L., You, P.C., Wu, D.M., Wang, M.H. and Wu, R.L. (2021) Effects of Yihuang Decoction on Vaginal Microecology and Local Immunity in Patients with Cervical High-Risk HPV Infection. Strait Pharmacy, 33, 93-95.

[11] Lin, R.R., Liu, J., Qiu, D.W. and Zeng, J.P. (2021) Changes of Vaginal Microecology in Patients with Different Degrees of Cervical Lesions and Its Relationship with HPV-DNA. Laboratory Medicine and Clinical, 18, 2866-2869.

[12] Rong, K. (2021) Analysis of Group B Streptococcus Infection and Vaginal Microecology in Pregnant Women with Term Premature Rupture of Membranes. China Maternal and Child Health, 36, 4703-4706.

[13] Huang, L.P. (2021) Vaginal Microecology in Assisted Reproductive Technology and Its Impact on Pregnancy Outcomes. Medical Equipment, 34, 72-73.

[14] He, M. (2021) Study on the Correlation between Vaginal Microecology, Human Papillomavirus Infection and Cervical Intraepithelial Neoplasia. China Journal of Health Inspection, 31, 2174-2176.

[15] Luo, Y.Y., Luo, X.W., Luan, F., Fu, L.H., Lin, Y.Y. and Qi, Q.P. (2021) Correlation between Vaginal Microecological Imbalance and Persistent High-Risk HPV Infection. Journal of Ningxia Medical University, 43, 811-815.

[16] Wu, Y.L., Huang, Y., Liu, Z. and Yang, Y. (2021) The Relationship between Vaginal Dysbiosis and High-Risk HPV Infection in Women of Childbearing Age. Hainan Medicine, 32, 2088-2090.

[17] Zhao, L., Cai, Z.W., Li, X.L. and Wang, Z.M. (2021) Discussion on the Correlation between Pregnancy Outcome and Vaginal Microecology in IUI Patients with Ovulation Induction. Yunnan Medicine, 42, 362-364.

[18] Ma, X.H., Song, D. and Ding, Y.H. (2021) Correlation between Vaginal Microecological Changes and Cervical High-Grade Squamous Intraepithelial Lesions. Ningxia Medical Journal, 43, 690-693.

[19] Zhu, J.B., Pan, X.J. and Xu, Y.K. (2021) Analysis of Vaginal Microecology in Patients with High-Risk Human Papillomavirus Infection and Its Correlation with Cervical Lesions. China Maternal and Child Health, 36, 3705-3707.

[20] Ji, X.Y. (2021) Effects of Honghe Fujie Lotion Combined with Lactobacillus Vaginal Capsules on Vaginal Microecological Indexes and Lavage Fluid Cytokine Levels in Patients with Fungal Vaginitis. Clinical Medical Research and Practice, 6, 89-91.

[21] Li, H.Y., Ying, L.D. and Sun, A.H. (2021) The Relationship between High-Risk Human Papillomavirus Infection and Cervical Lesions and Abnormal Vaginal Microecology. China AIDS and STD, 27, 767-769.

[22] Zhou, W. and Zhou, L.L. (2021) The Clinical Value of Urinary Cystatin C Combined with Serum $\beta$-2 Microglobulin Level in the Diagnosis of Patients with Lupus 
Nephritis. Marker Immunoassay and Clinical, 28, 1185-1187, 1221.

[23] Zhao, T. and Zhou, Q.Y. (2021) Correlation between Vaginal Microecological Indicators and Intrauterine Adhesions. Chinese Journal of Family Planning, 29, 1475-1479.

[24] Chen, S.P. (2021) The Diagnostic Value of Serum Cystatin C and Urine Kidney Injury Molecule-1 Detection for Early Kidney Injury in Patients with Hypertensive Disorders of Pregnancy. China Maternal and Child Health, 36, 555-557.

[25] Wang, C., Dong, M.T., Xue, F.X., et al. (2018) Pay Attention to the Application of Vaginal Microecological Detection in the Diagnosis and Treatment of Vaginal Inflammation in Women. Chinese Journal of Laboratory Medicine, 41, 256-258. 